\title{
Methyl group reorientation under ligand binding probed by pseudocontact shifts
}

\author{
Mathilde Lescanne ${ }^{1} \cdot$ Puneet Ahuja ${ }^{2} \cdot$ Anneloes Blok $^{1} \cdot$ Monika Timmer $^{1} \cdot$ Tomas Akerud $^{2} \cdot$ Marcellus Ubbink $^{1}(\mathbb{C}$
}

Received: 29 December 2017 / Accepted: 26 May 2018 / Published online: 2 June 2018

(c) The Author(s) 2018

\begin{abstract}
Liquid-state NMR spectroscopy is a powerful technique to elucidate binding properties of ligands on proteins. Ligands binding in hydrophobic pockets are often in close proximity to methyl groups and binding can lead to subtle displacements of methyl containing side chains to accommodate the ligand. To establish whether pseudocontact shifts can be used to characterize ligand binding and the effects on methyl groups, the N-terminal domain of HSP90 was tagged with caged lanthanoid NMR probe 5 at three positions and titrated with a ligand. Binding was monitored using the resonances of leucine and valine methyl groups. The pseudocontact shifts (PCS) caused by ytterbium result in enhanced dispersion of the methyl spectrum, allowing more resonances to be observed. The effects of tag attachment on the spectrum and ligand binding are small. Significant changes in PCS were observed upon ligand binding, indicating displacements of several methyl groups. By determining the cross-section of PCS iso-surfaces generated by two or three paramagnetic centers, the new position of a methyl group can be estimated, showing displacements in the range of 1-3 $\AA$ for methyl groups in the binding site. The information about such subtle but significant changes may be used to improve docking studies and can find application in fragment-based drug discovery.
\end{abstract}

Keywords Isotope labeling $\cdot$ Pseudocontact shift $\cdot$ Methyl groups $\cdot$ NMR spectroscopy $\cdot$ Paramagnetic tag $\cdot$ Heat shock protein

Electronic supplementary material The online version of this article (https://doi.org/10.1007/s10858-018-0190-5) contains supplementary material, which is available to authorized users.

Marcellus Ubbink

m.ubbink@chem.leidenuniv.nl

Mathilde Lescanne

m.lescanne@chem.leidenuniv.nl

Puneet Ahuja

puneet.ahuja.1983@gmail.com

Anneloes Blok

a.blok@chem.leidenuniv.nl

Monika Timmer

m.timmer.3@chem.leidenuniv.nl

Tomas Akerud

Tomas.Akerud@astrazeneca.com

1 Leiden Institute of Chemistry, Leiden University, Einsteinweg 55, 2333 CC Leiden, The Netherlands

2 Structure, Biophysics \& Fragment-Based Lead Generation, Discovery Sciences, IMED Biotech Unit, AstraZeneca, Gothenburg, Sweden

\author{
Abbreviations \\ PCS Pseudocontact shift \\ HSQC Heteronuclear single quantum coherence \\ TROSY Transverse relaxation optimized \\ spectroscopy \\ CLaNP-5 Caged lanthanoid NMR probe 5 \\ ntd-HSP90 N-terminal domain of heat shock protein 90
}

\section{Introduction}

Fragment-based drug discovery (FBDD) has proven to be an effective method to develop medicinal drugs (Erlanson 2012). FBDD is based on finding very small molecules that bind to the target with a large average binding energy per heavy atom $(\sim 0.3 \mathrm{kcal} / \mathrm{mol} / \mathrm{heavy}$ atom $)$. Such fragments still have a low affinity and then need to be elaborated to molecules with more negative binding free energies, either by linking fragment hits or by growing them (Bohacek et al. 1996; Hajduk and Greer 2007). For the elaboration of hits into lead compounds FBDD depends heavily on structural analysis of fragment-target complexes by X-ray 
diffraction of crystals or NMR spectroscopy. The former technique is most commonly used but NMR is an alternative for structure determination and offers complementary information. Structure determination of the complex by NMR requires a complete NOE analysis of protein and ligand, which is tedious but can be used in cases where X-ray crystallography fails (Pellecchia et al. 2008). Other, less demanding methods are based on transferred NOEs, paramagnetic relaxation enhancements (PRE) or pseudocontact shifts (PCS) to obtain information about the ligand bound state while benefitting from the narrow linewidths of the ligand in the free state (Viegas et al. 2011; Guan et al. 2013; Jahnke et al. 2000, 2001).

We aim to investigate the possibilities of PCS to study ligand-protein interactions. PCS have been used before to obtain model of ligands bound to proteins (Guan et al. 2013; Tu and Gochin 1999; Saio et al. 2011; John et al. 2006). Before, we demonstrated that ligands that are in fast exchange between bound and free state can exhibit transferred PCS caused by lanthanoid tags on the protein, which can be used to determine a low-resolution model of the ligand in the binding site, provided that a structure of the protein is available and under the assumption that ligand binding does not result in backbone conformational changes. Fragments, as well as larger compounds often bind in hydrophobic pockets on the protein where they do not alter the positions of backbone atoms significantly. Methyl groups are often found in such pockets and, thus, are in direct contact with the ligand. They are prone to experience chemical shift changes due to changes in the chemical environment upon ligand binding, and may also more readily than backbone atoms show conformational changes due to the rotational freedom of side-chains (Wiesnerl and Sprangers 2015). In this way, they can help to accommodate ligand binding, enabling it to form optimal interactions. Therefore, we wondered whether such changes could be detected by using PCS. These shifts are caused by the interaction of the nuclear spin and the spin of unpaired electron(s) in a paramagnetic center. They depend on the distance between the spin and the center to the third power as well as on the orientation of the spin in the frame of the anisotropic component of the magnetic susceptibility of the unpaired electron(s) (Otting et al. 2010; Liu et al. 2014). With a probe that is rigid relative to the protein and a proper diamagnetic control, the PCS can be predicted and measured with very high accuracy and small changes in the location of the spin relative to the paramagnetic center can result in measurable PCS changes. Methyl PCS can be observed in sensitive 2D NMR spectra, potentially also on large systems by applying selective labelling in a deuterated background and by using TROSY-based experiments (Tugarinov et al. 2003; Tugarinov and Kay 2005; Sprangers et al. 2008).
Here, we describe the use of PCS as structural restraints to probe at the same time binding kinetics and structural changes of the protein ntd-HSP90 upon fragment binding. HSP90 is a target protein against cancer (Nagaraju et al. 2017) and its ATP binding site located in the N-terminal domain (ntd) is targeted for inhibition ( $\mathrm{Li}$ et al. 2012). HSP90 is a molecular chaperone essential to prevent client proteins from ubiquitin-proteasome system degradation. More than 200 client proteins of HSP90 have been identified, including oncoproteins (Murray et al. 2010). Therefore, HSP90 is a cancer-target protein and inhibitors have been found to bind the $\mathrm{N}$-terminal domain and/or the C-terminal domain (Den and Lu 2012). Several potent molecules are clinical candidates for cancer treatment through inhibition of the ATPase activity and FBDD has been successfully applied to HSP90, which led to a clinical trial (Murray et al. 2010). We find that ligand binding is only marginally affected by attaching the two-armed lanthanoid tag CLaNP-5 (Keizers et al. 2007, 2008) to ntd-HSP90 at three locations. Methyl group resonances show extensive chemical shift perturbations in the binding site, as well as further in the hydrophobic core of the protein. Several significant PCS changes are observed upon ligand binding, which can be interpreted as movements of the methyl groups of a few Ångström. These changes can be translated into structural restraints that may be used in ligand docking studies.

\section{Materials and methods}

\section{Sample preparation}

Three double cysteine mutants of the ntd-HSP90 were designed on the surface of the protein, S50C/D54C, A101C/ N105C and T149C/I187C (Lescanne et al. 2017). NtdHSP90 does not have any native cysteines. The protein was produced labelled with Leu- $\delta 1-\delta 2 / \mathrm{Val}-\gamma 1-\gamma 2-\left[{ }^{13} \mathrm{CH}_{3}\right]$ and purified and tagged with CLaNP5 according to a published protocol (Lescanne et al. 2017). CLaNP-5 was synthesized as described before (Keizers et al. 2007, 2008).

\section{NMR titration}

Ntd-HSP90 in $50 \mathrm{mM}$ Tris- $\mathrm{HCl}$ and $50 \mathrm{mM} \mathrm{NaCl}$ buffer, $\mathrm{pH}$ 7.7, was titrated with 4-(2-Fluorophenyl)-2-pyrimidinamine, 1, (Fig. 1), a 189 Da known ligand of ntd-HSP90 that was kindly provided by AstraZeneca (Göteborg, Sweden). Titrations were performed with three ntd-HSP90 mutants, S50C/D54C, A101C/N105C and T149C/I187C tagged with $\mathrm{Lu}^{3+}{ }_{-} \mathrm{CLaNP}-5$ or $\mathrm{Yb}^{3+}{ }_{-} \mathrm{CLaNP}-5$. The concentrations of S50C/D54C, A101C/N105C T149C/I187C were 20, 103 and $65 \mu \mathrm{M}$, respectively, for both diamagnetic and paramagnetic forms of the protein. Concentrations $(\mu \mathrm{M})$ of $\mathbf{1}$ for titrations 


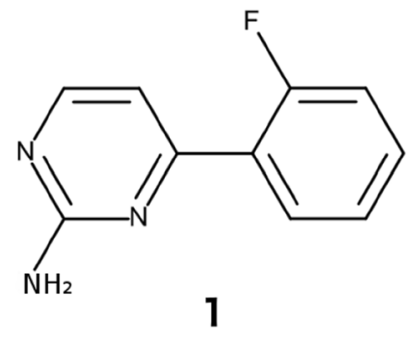

Fig. 1 Structure of 1 used to titrate ntd-HSP90

with S50C/D54C, A101C/N105C and T149C/I187C were $[0,39,59,88,132,198,296,444,667,1000],[0,20,40$, $121,364,1111]$ and $[0,40,89,200,442,665,1008,1897]$, respectively. The NMR sample volume was $595 \mu \mathrm{L}$ for all samples, and dilution was neglected, because the biggest volume of ligand solution added was $<5 \mu \mathrm{L} .{ }^{13} \mathrm{C}-{ }^{1} \mathrm{H}$ HSQC (Palmer et al. 1991; Kay et al. 1992; Schleucher et al. 1994) spectra were acquired at each titration point, on a Bruker Avance III $800 \mathrm{MHz}$ spectrometer, equipped with a cryogenically cooled TXI-probe head, operating at $298 \mathrm{~K}$. Spectra were processed with nmrpipe (Delaglio et al. 1995) using the exponential EM apodization function for analysis with TITAN (Waudby et al. 2016). A similar titration was performed with WT ntd-HSP90 observing the amide groups. Average CSP were calculated as $\left(\Delta \mathrm{H}^{2}+(\Delta \mathrm{C} / 10)^{2}\right)^{0.5}$ for methyl groups and $\left(\Delta \mathrm{H}^{2}+(\Delta \mathrm{N} / 6)^{2}\right)^{0.5}$ for amides. PCS were measured using ${ }^{1} \mathrm{H}$ and for calculations the geometrical average of the proton coordinates in methyl groups were used.

\section{Assignments}

Methyl groups assignments have been performed before with traditional through-backbone NMR techniques and confirmed by PARAssign (Skinner et al. 2013; Lescanne et al. 2017). PARAssign provided the stereo-specific assignment with high reliability for $14 \mathrm{Leu} / \mathrm{Val}$ methyl groups (Lescanne et al. 2017), based on PCS generated by CLaNP-5 attached at two distinct positions, S50C/D54C and A101C/N105C.

\section{Cross-section refinement}

A home-written python script was used to define possible locations of the bound methyl groups locations. PCS iso-surfaces were calculated for a grid of $15 \times 15 \times 15 \AA$, with 100 points per dimension and centered on the methyl group position in the crystal structure of free ntd-HSP90 [PDB entry $3 \mathrm{t} 0 \mathrm{~h}$ ( $\mathrm{Li}$ et al. 2012)]. Cross-sections of iso-surfaces from different tags were defined by finding the positions within the grid that matched the required PCS of all tags within an error of $0.02 \mathrm{ppm}$ (0.03 ppm in one case, see below).

\section{Q and Qa factors}

Q and Qa factors were used to quantify deviation between experimental and predicted data. Q and Qa were calculated according to Eqs. 1 and 2, respectively. Q is the usual measure for goodness of fit, Qa is, however, less sensitive to bias toward cases in which the predicted value is much larger than the observed one, as compared to the opposite case, in which the predicted value is much smaller than the observed one (Bashir et al. 2010). In cases of a good fit, $\mathrm{Qa} \approx 0.5 \mathrm{Q}$.

$Q=\sqrt{\frac{\sum\left(\delta_{P C S, i}^{\text {ped }}-\delta_{P C S, i}^{\text {exp }}\right)^{2}}{\sum\left(\delta_{P C S, i}^{\exp }\right)^{2}}}$

$Q a=\sqrt{\frac{\sum\left(\delta_{P C S, i}^{\text {pred }}-\delta_{P C S, i}^{\text {exp }}\right)^{2}}{\sum\left(\left|\delta_{P C S, i}^{\exp }\right|+\left|\delta_{P C S, i}^{\text {pred }}\right|\right)^{2}}}$

\section{Results}

\section{Tagging effects}

Ntd-HSP90 was tagged at three sites using the Caged Lanthanoid NMR probe \#5 (CLaNP-5), containing either $\mathrm{Lu}^{3+}$ as a diamagnetic control or $\mathrm{Yb}^{3+}$ as a paramagnetic center. The tagging sites, double mutants $50 \mathrm{C} / 54 \mathrm{C}, 101 \mathrm{C} / 105 \mathrm{C}$ and 149C/187C, have been described before (Lescanne et al. 2017). Assignments are shown in Fig. S1. Methyl ${ }^{13} \mathrm{C}-{ }^{1} \mathrm{H}$ HSQC spectra of WT and CLaNP-5 $\left(\mathrm{Lu}^{3+}\right)$ tagged mutants are very similar except for the resonance of a few methyl groups very close to the tags (Lescanne et al. 2017), indicating that the tags do not have large effects on the structure of the protein. A first comparison of the methyl group spectra of the paramagnetic and diamagnetic samples illustrates the increased dispersion of the resonances for the paramagnetic samples (Fig. 2), which has been noted before (Sattler and Fesik 1997). For example, a crowded spectral region with a width of $0.5 \mathrm{ppm}$ in the ${ }^{1} \mathrm{H}$ dimension in the spectrum of the diamagnetic sample, disperses over $1.0 \mathrm{ppm}$ for mutant $50 \mathrm{C} / 54 \mathrm{C}$ and up to $3.0 \mathrm{ppm}$ for mutant $101 \mathrm{C} / 105 \mathrm{C}$ in the spectrum of the paramagnetic sample. Increased dispersion is of interest for methyl group HSQC spectra, because the resonances are often more crowded than in amide HSQC spectra, in particular for Leu and Val methyl hydrogens and to a lesser extent methyl carbons. In principle, lanthanoids with larger paramagnetic effects $\left(\mathrm{Tm}^{3+}, \mathrm{Dy}^{3+}, \mathrm{Tb}^{3+}\right)$ provide even more dispersion but also cause considerable paramagnetic relaxation farther from the metal than in the case of 


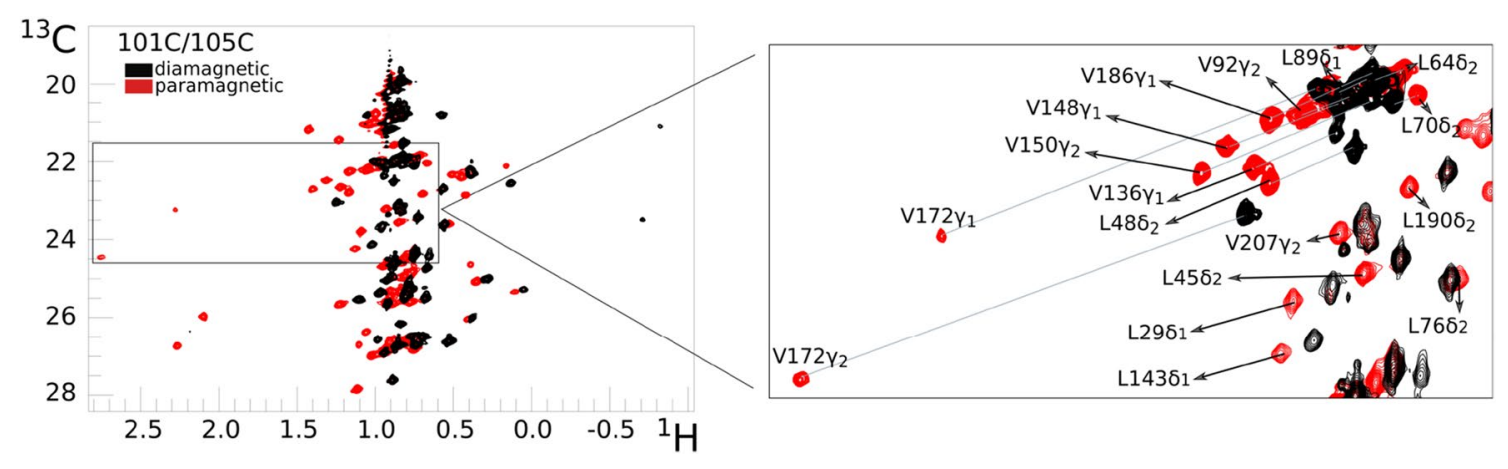

Fig. 2 Enhancement of spectral dispersion by PCS. An overlay is shown of Leu/Val methyl HSQC spectra of ntd-HSP90101C/105C tagged with CLaNP-5 loaded with $\mathrm{Lu}^{3+}$ (black contours) or $\mathrm{Yb}^{3+}$ (red contours). The inset shows a detail and the lines connect equivalent resonances

$\mathrm{Yb}^{3+}$. Thus, such lanthanoids are more appropriate to generate dispersion in spectra of bigger proteins.

Magnetic susceptibility $(\Delta \chi)$ tensors were refined previously using amide proton PCS (Lescanne et al. 2017). Methyl group PCS were predicted based on these tensors and the ligand-free structure [PDB entry $3 \mathrm{t} 0 \mathrm{~h}(\mathrm{Li}$ et al. 2012)] and compared to the experimental ones. For mutants $50 \mathrm{C} / 54 \mathrm{C}$ and $101 \mathrm{C} / 105 \mathrm{C}$ most predicted PCS fit the experimental values well (Fig. 3). Mutant 149C/187C shows a poorer fit but that is in line with the results for the amides (Fig. S2), which was attributed to the fact that the tag crosslinks two $\beta$-strands and appears to assume two conformations (Lescanne et al. 2017). To illustrate how differences between experimental and predicted PCS translate into differences in the expected locations of the nuclei in the protein, cross-sections of experimental PCS iso-surfaces for the different mutants were calculated (Fig. 4). The iso-surface identifies all locations around a paramagnetic center with a given PCS. The cross-sections are the overlap areas of two or three iso-surfaces from different tag locations. A cross-section was calculated such that its thickness reflects 0.02 ppm uncertainty. Thus, large cross-sections (Fig. 4a) indicate a weak PCS gradient, as is observed far from the paramagnetic centers. Thin crosssections (Fig. 4b) report on a steep PCS gradient, closer to the paramagnetic center or close to where the PCS changes sign. Cross-sections for the iso-surfaces of pairs of mutants are shown in Fig. 4, panels A and B, for the methyl groups from residues Leu70 and Val150. The predicted PCS of these methyl groups match the experimental PCS within $0.02 \mathrm{ppm}$. The methyl groups observed in the crystal structure are located at the intersection of the two cross-sections, i.e., at the position that matches the experimental PCS for all three mutants within $0.02 \mathrm{ppm}$. For some methyl groups the discrepancy between experimental PCS and PCS predicted on the basis of the crystal structure is larger than $0.02 \mathrm{ppm}$. For instance, the experimental PCS of Val136 $\gamma 1$ and $\gamma 2$ are -1.03 and $-1.12 \mathrm{ppm}$, (a)

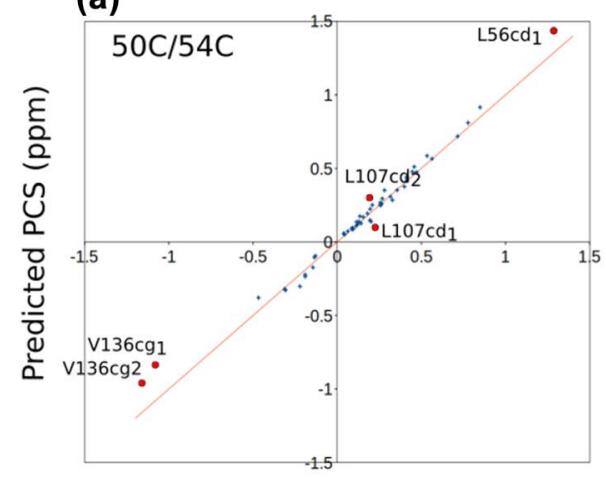

(b)

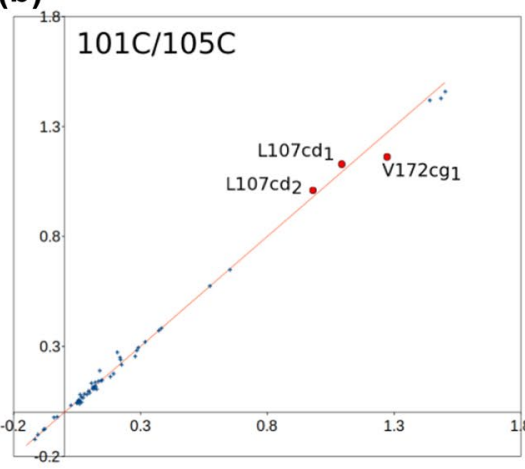

Experimental PCS (ppm) (c)

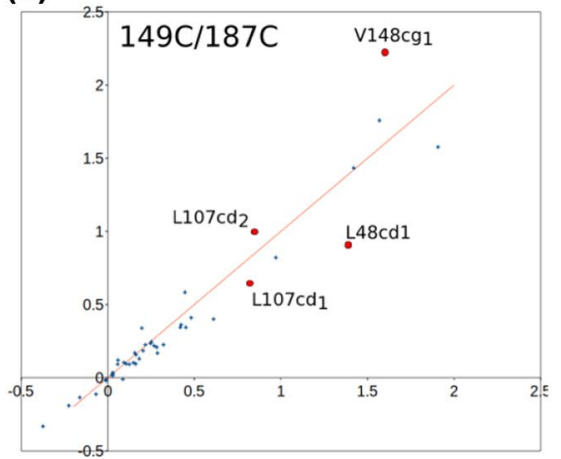

Fig. 3 Prediction of methyl group PCS. The PCS for the Leu/Val methyl groups of ntd-HSP90 were predicted using the published amide based $\Delta \chi$ tensor parameters (Lescanne et al. 2017) and the structure with PDB entry 3t0h (Li et al. 2012) and plotted against the experimental PCS. No fitting was performed. a For mutant $50 \mathrm{C} / 54 \mathrm{C}$ the $\mathrm{Q}$ factor (Eq. 1) is $0.14\left(\mathrm{Q}_{\mathrm{a}}=0.07\right.$, Eq. 2) b For mutant $101 \mathrm{C} / 105 \mathrm{C} \mathrm{Q}=0.05\left(\mathrm{Q}_{\mathrm{a}}=0.025\right)$. c For mutant $149 \mathrm{C} / 187 \mathrm{C} \mathrm{Q}=0.24$ $\left(\mathrm{Q}_{\mathrm{a}}=0.12\right)$. The red line represents a perfect correlation 

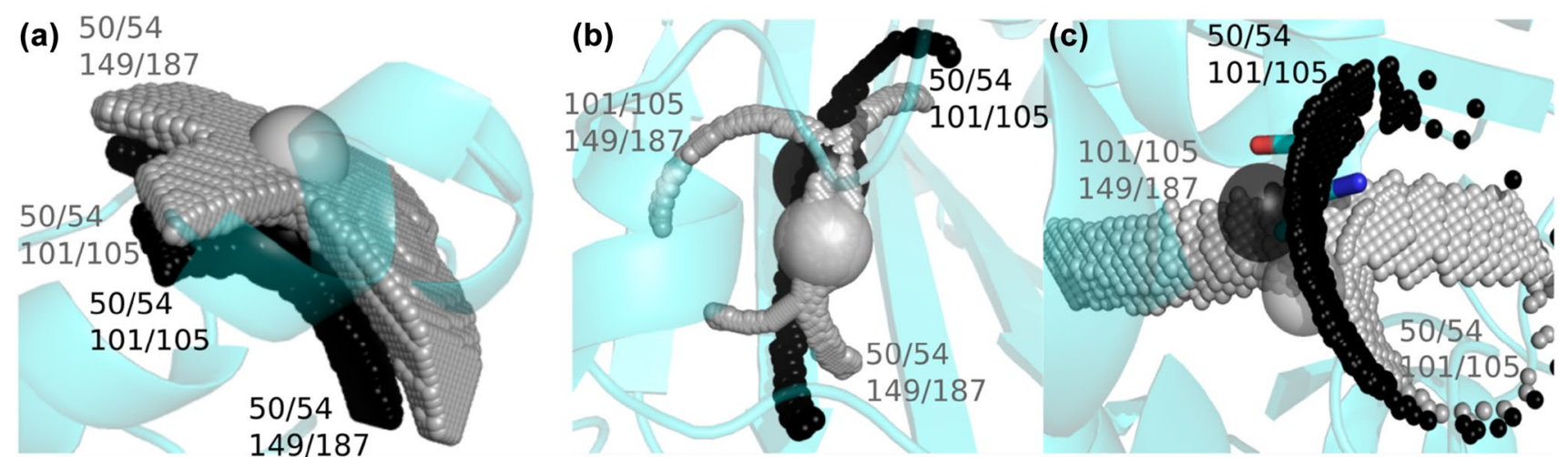

Fig. 4 Cross-sections of iso-surfaces of experimental free PCS, with the free structure $3 \mathrm{t} 0 \mathrm{~h}$. Large grey and black spheres represent the crystal structure locations of methyl groups, centred on the carbon methyl group with radius of $1 \AA$, for Val $\gamma 1 /$ Leu $\delta 1$ and Val $\gamma 2 /$ Leu 2 , respectively. Grey and black spheres represent the experimental PCS cross-sections for Val $\gamma 1 /$ Leu $\delta 1$ and Val $\gamma 2 / L e u \delta 2$, respectively for the free ntd-HSP90. Each cross-section was calculated using $0.02 \mathrm{ppm}$ error on the PCS, for a cubic grid with sides of 50 points over $5 \AA$, centred on the methyl group of interest. a Leu70 methyl groups. The two grey and black areas are cross-sections from mutant $50 \mathrm{C} / 54 \mathrm{C}$ with mutant $101 \mathrm{C} / 105 \mathrm{C}$ and mutant $50 \mathrm{C} / 54 \mathrm{C}$ with $149 \mathrm{C} / 187 \mathrm{C}$ for

respectively, and the predicted values show deviations of $0.19(-0.84 \mathrm{ppm})$ and $0.16 \mathrm{ppm}(-0.96 \mathrm{ppm})$ for mutant $50 \mathrm{C} / 54 \mathrm{C}$ for $\gamma 1$ and $\gamma 2$, respectively. Because of the strong PCS gradient, these large PCS deviations translate in only a small displacement of about $0.6 \AA$ as compared to the crystal structure (Fig. 4c). In the case of the mutant $149 \mathrm{C} / 187 \mathrm{C}$, the experimental PCS of Val136 $\gamma 1$ and $\gamma 2$ equal 0.27 and 0.27 and the deviations from the predicted values are only $0.03(0.23 \mathrm{ppm})$ and $0.05 \mathrm{ppm}(0.22 \mathrm{ppm})$, respectively, yet these translate into sizeable displacements of $1.5 \AA$, due to the weak PCS gradient at this position. This remarkable difference is visualized in Fig. S3. Thus, these findings demonstrate that deviations larger than the measurement error of PCS (usually $0.02 \mathrm{ppm}$ or less) can be caused by very subtle differences in structure, whereas small deviations may still reflect a more sizeable mismatch between observed PCS and the structure used for PCS prediction. The PCS gradient at the methyl group position can be used to evaluate the structural relevance for observed differences between experimental and predicted PCS. To establish to what degree the uncertainty in the $\Delta \chi$ tensor parameters affects the iso-surfaces, the $\Delta \chi$ tensors were also calculated using the methyl PCS as input, rather than the amide PCS. These independent data report on the same tensor, so differences are a measure for the uncertainty. The fitting involves eight parameters that are not completely uncorrelated, so slightly different solutions can be found. It was found that the difference are indeed small (Fig. S4). The effect on the calculated iso-surfaces only is significant for residues with a steep PCS gradient, Fig. S5.
Leu70 $\delta 1$ and $\delta 2$, respectively. b Val150 methyl groups. The grey areas are the $\gamma 1$ cross-sections from mutant $101 \mathrm{C} / 105 \mathrm{C}$ with mutant $149 \mathrm{C} / 187 \mathrm{C}$ and from mutant $50 \mathrm{C} / 54 \mathrm{C}$ with $149 \mathrm{C} / 187 \mathrm{C}$, the black area is the $\mathrm{V} 150 \gamma 2$ cross-section for mutant $50 \mathrm{C} / 54 \mathrm{C}$ with mutant $101 \mathrm{C} / 105 \mathrm{C}$. c Val136 methyl groups. In black spheres the PCS isosurfaces cross-section of mutant $50 \mathrm{C} / 54 \mathrm{C}$ with mutant $101 \mathrm{C} / 105 \mathrm{C}$ is shown for methyl $\gamma 2$. In grey spheres are the two PCS iso-surface cross-sections of mutant $50 \mathrm{C} / 54 \mathrm{C}$ with $101 \mathrm{C} / 105 \mathrm{C}$ and mutant $101 \mathrm{C} / 105 \mathrm{C}$ with mutant $149 \mathrm{C} / 187 \mathrm{C}$. Both crystal structure methyl groups are on the edge of the cross-section

For residue Leu107, the predicted PCS correlated poorly with the experimental ones for mutants $50 \mathrm{C} / 54 \mathrm{C}$ and $149 \mathrm{C} / 187 \mathrm{C}$. The predicted PCS are clearly distinct for the $\delta 1$ and $\delta 2$ methyl groups ( 0.072 and $0.252 \mathrm{ppm}$ for mutant $50 \mathrm{C} / 54 \mathrm{C}$ and 0.68 and $0.94 \mathrm{ppm}$ for mutant $149 \mathrm{C} / 187 \mathrm{C})$, whereas the experimental values are very similar $(0.221$ and $0.194 \mathrm{ppm}$ for mutant 50C/54C and 0.822 and $0.842 \mathrm{ppm}$ for mutant $149 \mathrm{C} / 187 \mathrm{C})$. The similarity of the two values suggests a kind of averaging. The linewidth of the resonances in the diamagnetic sample also suggests a form of exchange. To establish whether population of more than one rotamer explains the deviating PCS, the populations of each of the three rotamers were determined. Each rotamer was modelled in the structure with Pymol (DeLano 2009) (ignoring steric clashes) and the PCS were predicted. The best fit was found for an exchange between two rotamers populated at 53 and $47 \%$, with the third rotamer not being populated, Fig. 5 . The Qa fit quality factor (see Eq. 2) for Leu107 drops from 0.14 to 0.03 when predicted PCS are calculated as a combination of rotamer $2\left(+0^{\circ}\right)$ and rotamer $3\left(+120^{\circ}\right)$, for mutants $50 \mathrm{C} / 54 \mathrm{C}$ and $149 \mathrm{C} / 187 \mathrm{C}$. For mutant $101 \mathrm{C} / 105 \mathrm{C}$, rotamer 2 fits the data well and admixture of rotamer 3 reduces the fit quality. However, Leu107 is located very close to the tag in this mutant, and near a reported flexible region (Didenko et al. 2012), so either the methyl group location or the exchange populations of rotamers could be influenced by the CLaNP tag. It is interesting that the PCS seem to provide evidence for rotamer exchange and can be used to estimate populations. Such dynamics is not obvious from the chemical shift. Definitive evidence, however, would require further 


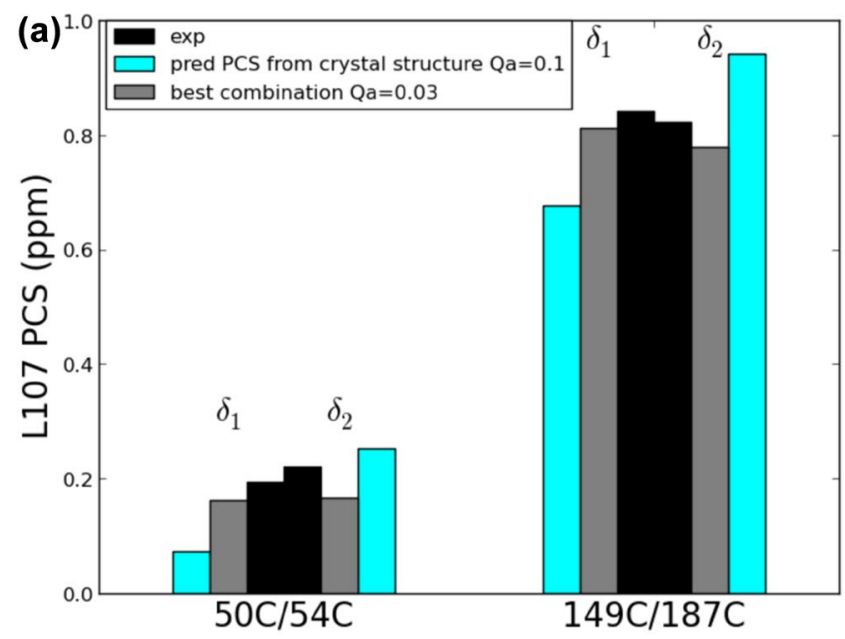

(b)

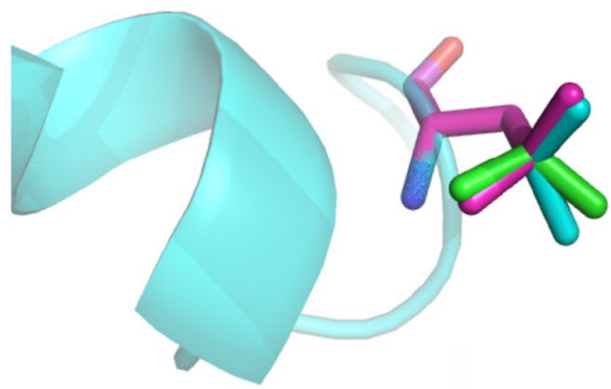

Fig. 5 a Experimental (black) and predicted PCS for the two methyl groups of L107 for rotamer 2, observed in the crystal structure [PDB entry $3 \mathrm{t} 0 \mathrm{~h}$ ( $\mathrm{Li}$ et al. 2012)] in cyan and for the combination of rotamer 2 and 3 in grey, for the two mutants $50 \mathrm{C} / 54 \mathrm{C}$ and $149 \mathrm{C} / 187 \mathrm{C}$. b Residue L107. The rotamer found in the crystal structure is shown in

experiments, which were beyond the scope of this work. No other methyl groups were found to be experiencing the same phenomenon.

\section{Ligand titration}

First, ntd-HSP90 mutants tagged with diamagnetic CLaNP-5 were titrated with the weakly binding ligand 1 . Three regions of the protein exhibit chemical shift perturbations (CSP) upon titration (Fig. 6), revealing a localized binding site, in a cleft of the protein, but with small CSP being observed relatively far away in the core of the protein, Fig. 7. The affected regions are similar for the three mutants (Fig. 6), suggesting that the tag at different locations does not alter the binding location. Moreover, most peaks show similar CSP directions in the two-dimensional HSQC spectra (Fig. S6), indicating similar changes in the chemical environment of the methyl groups upon ligand binding. An exception is the resonance for Leu103 $\delta 1$, for which the CSP is not the same for all mutants. The discrepancy is largest for mutant $101 \mathrm{C} / 105 \mathrm{C}$, in which the Leu is very close to the tag, being located in between the two engineered Cys residues. Thus, the tag at this position appears to have some effect on ligand binding. A titration of the WT ntd-HSP90 in which the amide groups were observed was also performed. The results are in line with the methyl titrations, with the largest effects near the binding cleft, in particular in the long $\alpha$-helix that lines the binding site. Also for the amides, smaller effects are seen far from the binding site, suggesting that binding effects are transmitted into the rest of the protein (Fig. 7). cyan sticks. Rotamers 1 and 3 are in green and magenta, respectively. The rotamers were generated with Pymol (DeLano 2009). Note that the idealized, generated rotamers shows slightly different angles compared to the crystal structure rotamer

\section{Binding parameters}

The dissociation constant $\mathrm{K}_{\mathrm{D}}$, as well as the dissociation rate constant $\left(\mathrm{k}_{\mathrm{OFF}}\right)$ were calculated with TITAN software (Waudby et al. 2016) using a 1:1 binding model $(\mathrm{A}+\mathrm{B} \leftrightarrows \mathrm{A}-\mathrm{B})$. TITAN fits both the equilibrium constant $\left(\mathrm{K}_{\mathrm{D}}\right)$ and the dissociation rate constant $\left(\mathrm{k}_{\mathrm{OFF}}\right)$ on the basis of the CSP and line broadening. A global fit of the resonances of five methyl groups (Table S1) was performed and then one at the time was taken out and the fit repeated. The WT $\mathrm{K}_{\mathrm{D}}$ was calculated using a titration performed on the ${ }^{15} \mathrm{~N}$ labelled protein, on the basis of four amide protons (Table S1). The ranges of values obtained in this way are reported in Table 1 and provide a more realistic error range than the fit error. Differences are observed between the different mutants, suggesting that the affinity is influenced by the tags to some degree, in particular for mutant $149 \mathrm{C} / 187 \mathrm{C}$. $\mathrm{K}_{\mathrm{D}}$ values of 200 and $150 \mu \mathrm{M}$ were used for the calculation of the $100 \%$ bound state CSP for mutant $50 \mathrm{C} / 54 \mathrm{C}$ and $101 \mathrm{C} / 105 \mathrm{C}$ respectively. For these two mutants, a $50 \mu \mathrm{M}$ variation of the $K_{D}$ results in a change of the extrapolated CSP of $0.01 \mathrm{ppm}$ at most, half the error used for the further calculations. For mutant $149 \mathrm{C} / 187 \mathrm{C}$ a $\mathrm{K}_{\mathrm{D}}$ of $50 \mu \mathrm{M}$ was used to extrapolate the CSP.

\section{Methyl group re-orientation}

The titrations were repeated with ntd-HSP90 tagged with CLaNP-5 $\left(\mathrm{Yb}^{3+}\right)$. The binding characteristics were the same as for the diamagnetic sample. To obtain the PCS for the ligand bound state, the maximal CSP, representing 

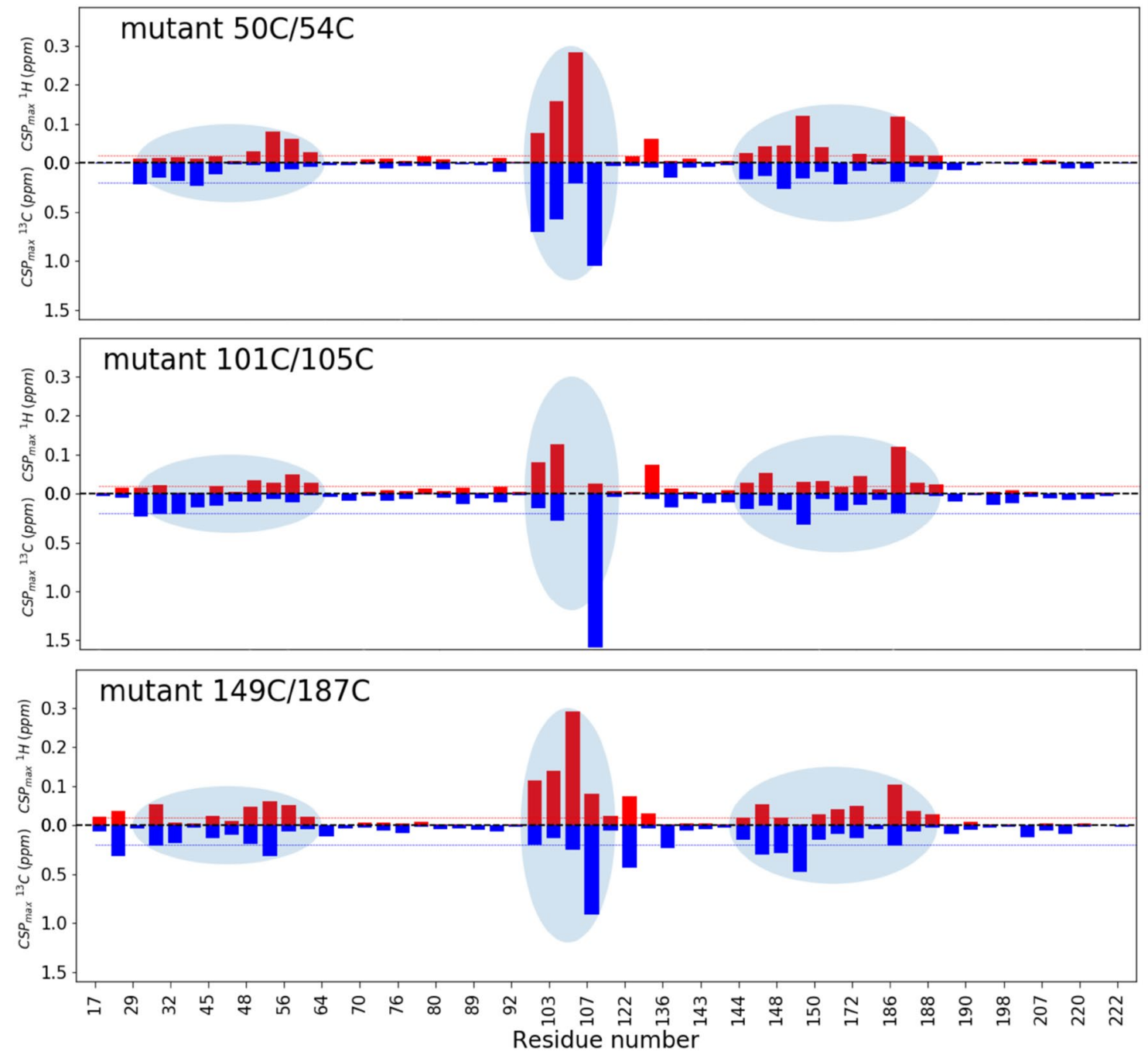

Fig. 6 Ligand binding. ICSPI are plotted for methyl group resonances upon binding of 1 . The ${ }^{1} \mathrm{H}$ and ${ }^{13} \mathrm{C} \mathrm{CSP}$ are shown in red and blue bars, respectively. The CSP have been extrapolated to the $100 \%$

ntd-HSP90 fully saturated with $\mathbf{1}$, were calculated for both diamagnetic and paramagnetic proteins by extrapolation from the CSP data in the last point of the titration, by using the $\mathrm{K}_{\mathrm{D}}$ values. The extrapolated CSP were subtracted to obtain the PCS of the bound state. Ligand binding causes changes in some PCS up to $0.1 \mathrm{ppm}$, Fig. 8. All PCS values are provided in Table $\mathrm{S} 2$. Methyl groups exhibiting significant $\triangle \mathrm{PCS}$, larger than $0.04 \mathrm{ppm}$ for at least two of the paramagnetic centers, were selected for further analysis, comprising $\mathrm{L} 48_{\delta 2}, \mathrm{~L} 103_{\delta 1}, \mathrm{~L} 107_{\delta 2}$ and $\mathrm{V} 136_{\gamma 1}$ (Table 2). These residues surround the binding site of ntd-HSP90, Fig. 7. Changes in PCS could be caused by changes in the position of the methyl groups due to interactions with the ligand, by changes in the tag position or orientation or by a combination of both effects. The $\Delta \chi$ tensors were calculated from the PCS of the complex of ntd-HSP90 and 1 (Table S3) bound state. The red and blue lines mark 0.02 and $0.2 \mathrm{ppm}$, respectively. The light blue ovals highlight the most perturbed methyl groups

and back-predicted PCS were compared with those of the free protein. The correlation is very good, with $\mathrm{Q}$ a values of $0.03,0.04$ and 0.16 for mutants $50 \mathrm{C} / 54 \mathrm{C}, 101 \mathrm{C} / 105 \mathrm{C}$ and 149C/187C, respectively (Fig. S7). The Qa values are very similar to those found between the experimental PCS and the back-calculated PCS after tensor refinement (Lescanne et al. 2017). Thus, any change of the $\Delta \chi$ tensors is within the precision of its parameters. The fact that the largest PCS changes map to methyl groups located in the binding site also provides evidence that not a tensor change but movement of the methyl groups is the cause of the PCS changes. Under that assumption, the following calculations were done. We wondered whether the PCS can provide information about the distance range of the rearrangement as well as the new location of the methyl groups. Using the PCS of the bound form, the possible new positions can be calculated 
Fig. 7 CSP map for binding of 1 to ntd-HSP90. The smaller spheres represent the Leu and Val methyl groups, coloured in a gradient of white to blue for increasing CSP as observed in the titration of mutant $50 \mathrm{C} / 54 \mathrm{C}$ tagged with CLaNP-5 $\left(\mathrm{Lu}^{3+}\right)$. The larger spheres represent the amide nitrogens that were observed in a titration of WT ntd-HSP90, coloured according the average amide CSP (from white to red). The residues L48, L103, L107 and V136, harboring a methyl group with $|\Delta \mathrm{PCS}|>0.04 \mathrm{ppm}$ for at least two mutants, are shown in yellow sticks. The structure is taken from PDB entry $3 \mathrm{t} 0 \mathrm{z}$. The ATP ligand observed in that structure is indicated by semi-tranparent, cyan sticks to indicate the binding site

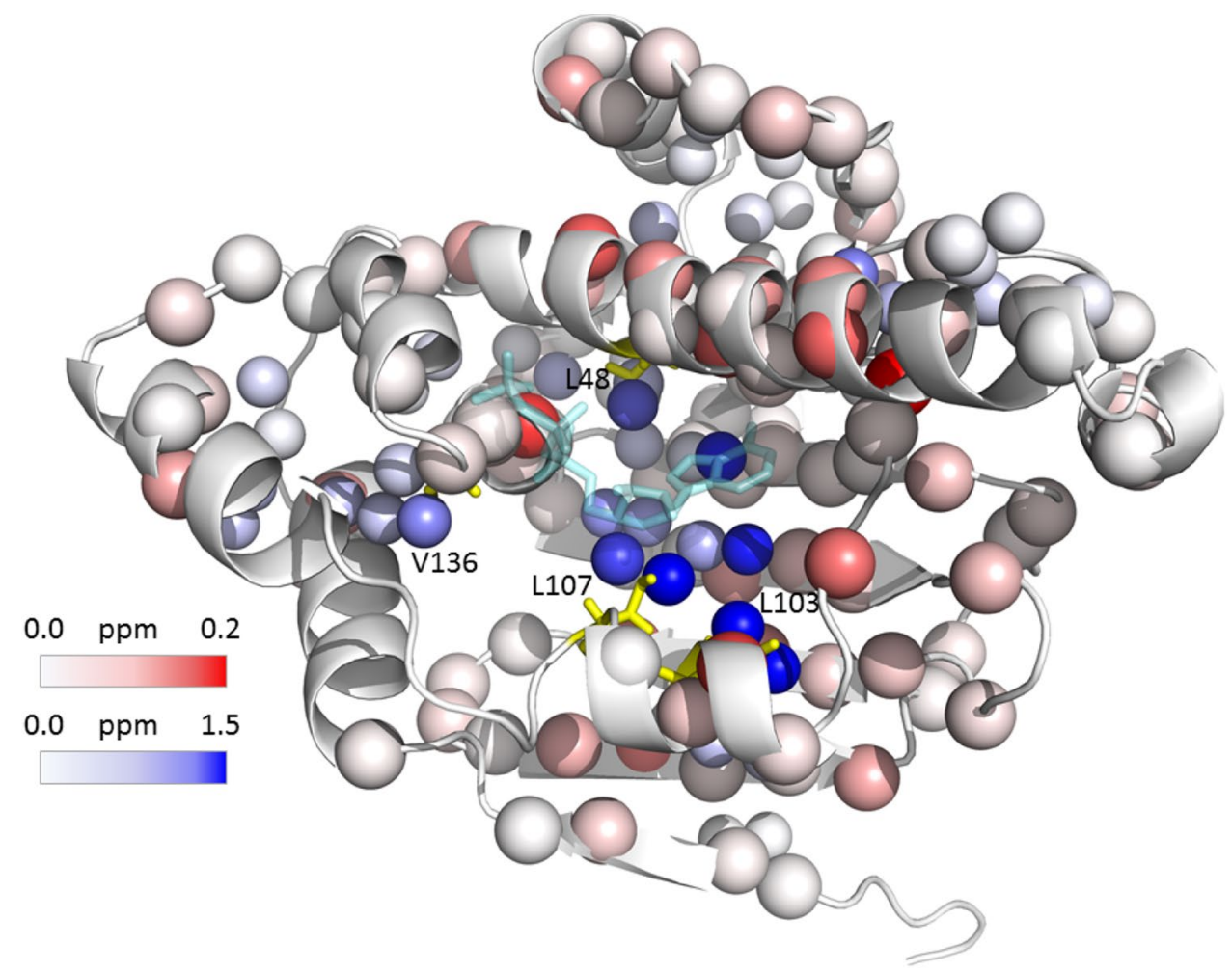

Table 1 Parameters for binding of 1 to ntd-HSP90 derived from the titrations with TITAN software, for the WT and three mutants

\begin{tabular}{lll}
\hline & $\mathrm{K}_{\mathrm{D}}(\mu \mathrm{M})$ & $\mathrm{k}_{\mathrm{OFF}}\left(\mathrm{s}^{-1}\right)$ \\
\hline $\mathrm{WT}$ & $160-220$ & $1400-1800$ \\
$50 \mathrm{C} / 54 \mathrm{C}$ & $195-238$ & $1500-2300$ \\
$101 \mathrm{C} / 105 \mathrm{C}$ & $140-171$ & $1075-1300$ \\
$149 \mathrm{C} / 187 \mathrm{C}$ & $41-45$ & $1300-1900$ \\
\hline
\end{tabular}

as an iso-surface of the new PCS around the lanthanoid. By determining the cross-sections of such iso-surfaces from two or even three mutants, the location space can be reduced, as was shown above for the free protein.

All cross-sections for the ligand bound state could be determined using an uncertainty of $0.02 \mathrm{ppm}$, except for Leu107, for which the uncertainty had to be raised to $0.03 \mathrm{ppm}$ to find a cross-section. The cross-sections are shown in Fig. 9 in grey $(\gamma 1 / \delta 1)$ and black $(\gamma 2 / \delta 2)$ for the PCS of free protein and in yellow $(\gamma 1 / \delta 1)$ and orange $(\gamma 2 /$ $\delta 2$ ) for the PCS observed for the bound state. For Leu 48 , iso-surfaces could be calculated for mutants $50 \mathrm{C} / 54 \mathrm{C}$ and $101 \mathrm{C} / 105 \mathrm{C}$ and the cross-sections are shown for the $\delta 1$ and $\delta 2$ methyl groups, Fig. 9a. The conformation of the Leu in the free protein is shown in sticks. The Leu methyl groups need to move at least $1.3 \AA$ to move into the cross-section area of the bound state. Similarly, Fig. $9 \mathrm{~b}$ shows the crosssections for Leu103. In this case, data from all three mutants result in only two possible positions for the Leu that can match simultaneously the PCS of both methyl groups to the experimental ones. The center of the triple mutant crosssection area for the $\delta 1$ methyl is shifted by $3 \AA$ upon binding of the ligand, whereas for the $\delta 2$ methyl it does not change, which could indicate that the sidechain rotates. Similarly, the $\gamma 1$ methyl group of Val136 experiences a significant change in PCS, whereas the $\gamma 2$ methyl group does not. The crosssection center of the $\gamma 1$ methyl group for mutant $50 \mathrm{C} / 54 \mathrm{C}$ with mutant $101 \mathrm{C} / 105 \mathrm{C}$ shifted $1.2 \AA$ from the position in the free protein. In Fig. 9c, it can be that the cross-section area for the bound state (yellow) has moved relative to the one for the free state (light gray). For Leu107, the two isosurfaces, from mutants $50 \mathrm{C} / 54 \mathrm{C}$ and $149 \mathrm{C} / 187 \mathrm{C}$ are similar because the two tensors happen to be nearly parallel (Lescanne et al. 2017). Consequently, the cross-section is a curve area rather than a line like in the cases discussed above (Fig. S8). This cross-section area in the bound state is at a large distance from the conformation of Leu107 in the crystal structure of the free protein, the minimal distance between the $\delta 2$ methyl group and the cross-section being more than $5 \AA$, while it is only $0.6 \AA$ for the free state. Thus, a large change in position is suggested by the observed change in the PCS. In different crystal structures of ntd-HSP90, Leu 107 is found in very different orientations, with $3.0 \AA$ between $\mathrm{C} \alpha$ atoms in the structure of the free protein [PDB entry 3t0h ( $\mathrm{Li}$ et al. 2012)] and the one with ntd-HSP90 bound to a close analogue of $\mathbf{1}$ [PDB entry: $2 \mathrm{xdk}$ 


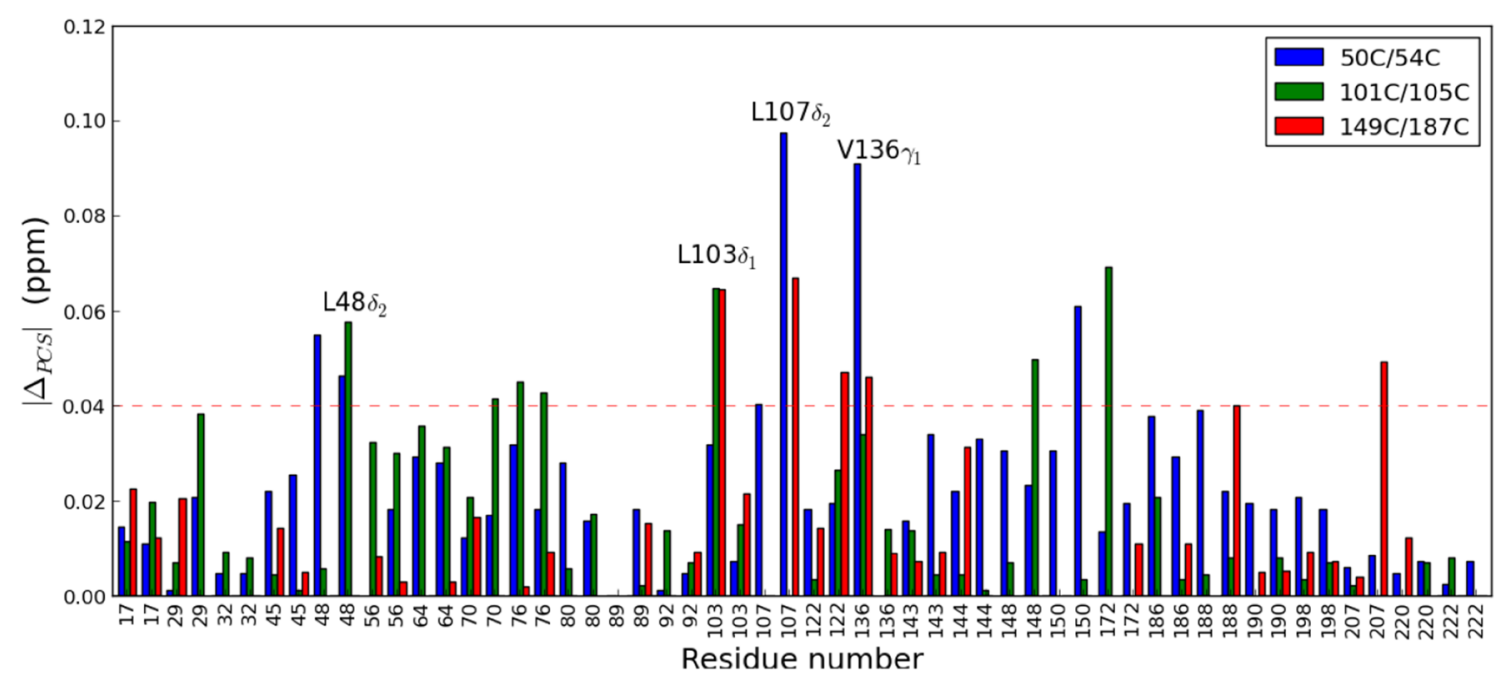

Fig. 8 PCS changes upon ligand binding. I $\triangle \mathrm{PCS} \mid$ for Leu/Val methyl groups for the bound state are shown as blue, green and red bars for the mutants $50 \mathrm{C} / 54 \mathrm{C}, 101 \mathrm{C} / 105 \mathrm{C}$ and $149 \mathrm{C} / 187 \mathrm{C}$, respectively. The red dashed line represents the threshold of $0.04 \mathrm{ppm}$, based on the

Table 2 Significant $\triangle \mathrm{PCS}$ (ppm). Methyl groups for which two $|\triangle \mathrm{PCS}|>0.04 \mathrm{ppm}$ are listed

\begin{tabular}{lrll}
\hline & $50 \mathrm{C} / 54 \mathrm{C}$ & $101 \mathrm{C} / 105 \mathrm{C}$ & $149 \mathrm{C} / 187 \mathrm{C}$ \\
\hline $\mathrm{L}_{48}$ & -0.046 & -0.058 & No data \\
$\mathrm{L}_{\delta 2}$ & -0.032 & -0.065 & 0.064 \\
$\mathrm{~L}_{\delta 1}$ & 0.097 & No data & 0.067 \\
$\mathrm{~V} 136_{\gamma 1}$ & 0.091 & 0.039 & -0.046 \\
\hline
\end{tabular}

estimated error. The conservative maximum experimental error in the chemical shifts is estimated to be $0.02 \mathrm{ppm}$. The propagated error in $\triangle \mathrm{PCS}$ is thus $0.04 \mathrm{ppm}$

(Murray et al. 2010)]. This observation suggests that Leu107 is very sensitive to ligand binding. Therefore, the observed change in methyl group position is plausible, although it should be treated with caution because of the high degree of correlation between the two $\Delta \chi$ tensors involved. The data for mutant $101 \mathrm{C} / 105 \mathrm{C}$ cannot be used in this case because the tag is too close to this Leu 107.

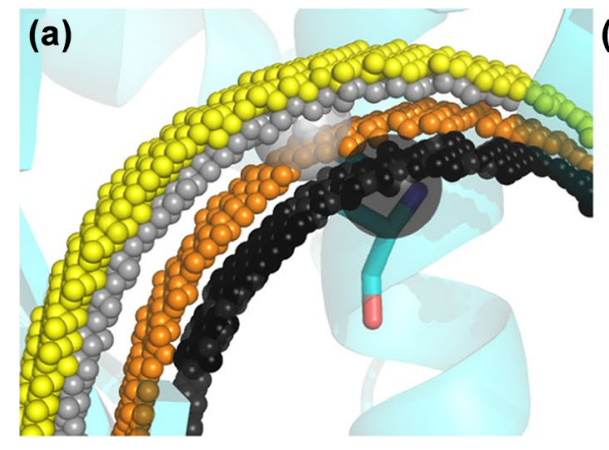

(b)

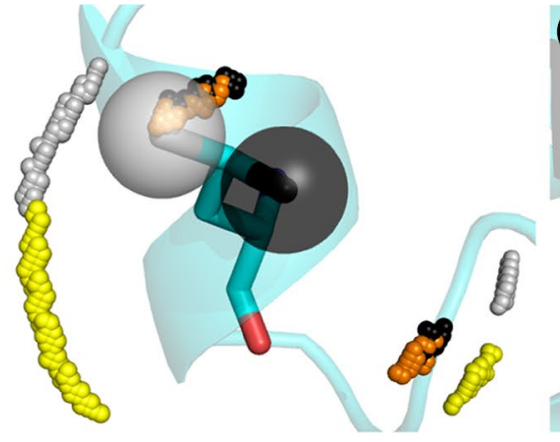

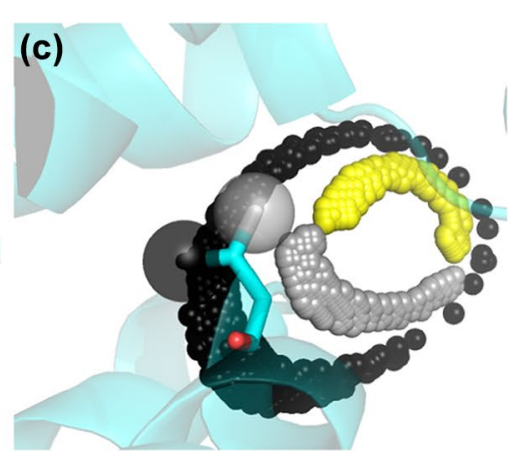

Fig. 9 Positions for methyl groups in the ntd-HSP90 bound to $\mathbf{1}$. Small spheres represent PCS iso-surfaces cross-sections in the free state, grey for Val $\gamma 1 /$ Leu $\delta 1$ and black for Val $\gamma 2 /$ Leu $\delta 2$, and in the bound state, yellow for Val $\gamma 1 /$ Leu $\delta 1$ and orange for Val $\gamma 2 /$ Leu $\delta$. The residues as found in the crystal structure of the free protein (PDB entry $3 \mathrm{t} 0 \mathrm{~h}$ ) are shown in cyan sticks, with the methyl groups in the analogous colors shown as $0.7 \AA$ radius spheres centred on the carbon methyl. a Leu48, with cross-sections for PCS from mutants 50C/54C and $101 \mathrm{C} / 105 \mathrm{C}$. The minimal distance from the crystal structure conformation to the free cross-sections is $0.8 \AA(\delta 1)$ and $0.6 \AA(\delta 2)$, and to the bound cross-sections is $1.3 \AA(\delta 1)$ and $1.2 \AA(\delta 2)$. b Leu103, the yellow and orange spheres represent the triple cross-sections of mutant $50 \mathrm{C} / 54 \mathrm{C}$ with $101 \mathrm{C} / 105 \mathrm{C}$ and $149 \mathrm{C} / 187 \mathrm{C}$. The grey and black spheres represent the triple-mutant cross-sections for the free state, at a distance of $1.6 \AA(\delta 1)$ and $2.3 \AA(\delta 2)$ from the crystal structure positions. Note that the orange and black region overlap whereas the grey and yellow are bordering, suggesting that methyl $\delta 1$ moves, whereas $\delta 2$ does not. c Val136, the grey and yellow small spheres represent the triple cross-section for methyl group $\gamma 1$ in free and bound state respectively. The small black spheres represent the free double cross-section between mutant $50 \mathrm{C} / 54 \mathrm{C}$ and $101 \mathrm{C} / 105 \mathrm{C}$ of group $\gamma 2$. The orange cross-section is not shown because the $\gamma 2$ methyl group showed insignificant PCS changes 
In conclusion, the small but significant changes in the PCS yield consistent information about the change in methyl group position that can be in the range of $1-5 \AA$. The isosurface approach yields a limited set of possible conformations, in particular in the case when data from three tag locations are available. These data could be translated into distance restraints, for example for ligand docking studies.

\section{Conclusions}

Methyl groups are widely used NMR probes but the applications of PCS to study them remain limited (Brewer et al. 2015). A straightforward application of paramagnetic tagging is the additional dispersion induced by PCS. Paramagnetic tags have already been used to disperse resonances of an intrinsically disordered protein, presenting a crowded amide proton spectrum (Gobl et al. 2016). Our data demonstrate again that additional dispersion is also relevant for folded, larger proteins that are being studied with methyl group NMR probes, because two-dimensional spectra usually show considerable crowding for methyl resonances in the ${ }^{1} \mathrm{H}$ region of $0.8-1.0 \mathrm{ppm}$, as was reported and used before (Sattler and Fesik 1997). Ytterbium was the lanthanoid of choice for a $25 \mathrm{kDa}$ protein. For larger proteins, lanthanoid of different paramagnetic 'strength' could be used to benefit from a high dispersion, while limiting loss of information because of PRE.

Furthermore, PCS were used to provide evidence for small-scale movements (1-3 $\AA$ ) of methyl groups upon ligand binding, by using a triangulation approach. This makes the PCS a powerful tool to observe re-arrangement of the side chains solely on the basis of 2D NMR spectra. Of course, assignments are required for the interpretation of such data. Recently, we have demonstrated that the same paramagnetic constructs used in the ligand titrations could be used to obtain partial assignments of the methyl groups (Lescanne et al. 2017). Thus, this application of PCS could complement the use of NOESY experiments, or indeed crystal structures, for structure determination of ligand-protein complexes. An advantage is that for measurement of PCS only low sample concentrations are required. It is clear that a single paramagnetic center is not sufficient to define a relevant area for the methyl group position in the bound state. With two paramagnetic centers and the use of both methyl groups of valine and/or leucine the positions can be approximated, because of the additional steric constraint that the two methyl groups must be at a distance of $2.5 \AA$. With three tags, the location becomes quite restrained, although two or three quite different positions may be found due to the shape of PCS iso-surfaces. Attention should be paid to the relative orientations of the susceptibility tensors used to generate the PCS. In case of tensors with parallel main axes, cross-intersections of iso-surfaces are less resolved or non-existing. When using PCS for methyl localization, it is important to realize that the PCS is highly anisotropic and falls off with the third power of the distance between the nucleus and the paramagnetic center. Localized, high PCS gradients make the PCS very sensitive to the methyl group position. That is an advantage but also a danger. The higher the PCS gradient, the larger the effect of any PCS error will be. The use of several tag positions can counteract this problem. However, this approach also demonstrated that positions predicted from PCS from two or three tags do not always match perfectly with those observed in the crystal state of the protein, even in the absence of ligand. The reasons are not clear. It could be that the average position of these methyl groups is slightly different in the solution state but it is also possible that (one of) the tags have subtle structural effects causing a small mismatch between PCS of the native and tagged protein variants. Consequently, to study the effects of ligand binding it is recommended to use the difference in PCS between free and bound states to derive distance restraints for methyl movement, as was discussed above. To obtain reliable PCS the use of a probe that is rigid relative to the protein is important. The high rigidity of two-armed CLaNP-5 enables the measurement of accurate PCS and the prediction of the tensors parameters accurately. The choice of the lanthanoid determines the optimal region. Nuclei too close to the tag will experience PRE, nuclei too far experience small changes in PCS upon displacement. With $\mathrm{Yb}^{3+}$ used here, distances between nucleus and tag in the range of $50 \AA$ yielded PCS changes of $0.04-0.1 \mathrm{ppm}$ that appear to match with displacements of $1-3 \AA$. The ability to observe and characterize small but significant changes in the methyl group positions can potentially also be applied to large proteins, because it is well-established that with deuteration methyl groups can be detected in very large systems (Saio et al. 2014; Kerfah et al. 2015). We also note that this approach is not limited to ligands that are in fast exchange. As long as assignments are available for free and bound states of the protein, PCS can also be obtained in slow-exchange systems.

Funding The research leading to these results has received funding from the People Programme (Marie Curie Actions) of the European Union's Seventh Framework Programme FP7/2007-2013 under Research Executive Agency Grant 317127 (pNMR).

Open Access This article is distributed under the terms of the Creative Commons Attribution 4.0 International License (http://creativeco mmons.org/licenses/by/4.0/), which permits unrestricted use, distribution, and reproduction in any medium, provided you give appropriate credit to the original author(s) and the source, provide a link to the Creative Commons license, and indicate if changes were made. 


\section{References}

Bashir Q, Volkov AN, Ullmann GM, Ubbink M (2010) Visualization of the encounter ensemble of the transient electron transfer complex of cytochrome c and cytochrome c peroxidase. J Am Chem Soc 132:241-247. https://doi.org/10.1021/ja9064574

Bohacek, R.S., McMartin, C. \& Guida, W.C. The art and practice of structure-based drug design: a molecular modeling perspective. Med. Res. Rev. 16, 3-50 (1996)

Brewer KD et al (2015) Dynamic binding mode of a Synaptotagmin1-SNARE complex in solution. Nat Struct Mol Biol 22:555. https ://doi.org/10.1038/nsmb.3035

Delaglio F et al (1995) Nmrpipe-a multidimensional spectral processing system based on unix pipes. J Biomol NMR 6:277-293. https ://doi.org/10.1007/BF00197809

DeLano WL (2009) PyMOL molecular viewer: updates and refinements. Abstracts of Papers of the Am Chem Soc 238

Den RB, Lu B (2012) Heat shock protein 90 inhibition: rationale and clinical potential. Ther Adv Med Oncol 4:211-218. https://doi. org/10.1177/1758834012445574

Didenko T, Suarte MSA, Karagöz EG, Rüdiger SGD (2012) Hsp90 structure and function studied by NMR spectroscopy. Biochim Biophys Acta 1823:636-647. https://doi.org/10.1016/j.bbamc r.2011.11.009

Erlanson DA (2012) Introduction to fragment-based drug discovery. Top Curr Chem 317:1-32. https://doi.org/10.1007/128_2011_180

Gobl C et al (2016) Increasing the chemical-shift dispersion of unstructured proteins with a covalent lanthanide shift reagent. Angew Chem Int Ed 55:14847-14851. https://doi.org/10.1002/anie.20160 7261

Guan JY et al (2013) Small-molecule binding sites on proteins established by paramagnetic NMR spectroscopy. J Am Chem Soc 135:5859-5868. https://doi.org/10.1021/ja401323m

Hajduk PJ, Greer, J (2007) A decade of fragment-based drug design: strategic advances and lessons learned. Nat Rev Drug Discov 6:211-219. https://doi.org/10.1038/nrd2220

Jahnke W et al (2000) Second-site NMR screening with a spinlabeled first ligand. J Am Chem Soc 122:7394-7395. https://doi. org/10.1021/ja001241+

Jahnke W, Rudisser S, Zurini M (2001) Spin label enhanced NMR screening. J Am Chem Soc 123:3149-3150. https://doi. org $/ 10.1021 / \mathrm{ja} 005836 \mathrm{~g}$

John M, Pintacuda G, Park AY, Dixon NE, Otting G (2006) Structure determination of protein-ligand complexes by transferred paramagnetic shifts. J Am Chem Soc 128:12910-12916. https://doi. org/10.1021/ja063584z

Kay LE, Keifer P, Saarinen T (1992) Pure absorption gradient enhanced heteronuclear single quantum correlation spectroscopy with improved sensitivity. J Am Chem Soc 114:10663-10665. https://doi.org/10.1021/ja00052a088

Keizers PHJ, Desreux JF, Overhand M, Ubbink M (2007) Increased paramagnetic effect of a lanthanide protein probe by twopoint attachment. J Am Chem Soc 129:9292-9293. https://doi. org/10.1021/ja0725201

Keizers PHJ, Saragliadis A, Hiruma Y, Overhand M, Ubbink M (2008) Design, synthesis, and evaluation of a lanthanide chelating protein probe: CLaNP-5 yields predictable paramagnetic effects independent of environment. J Am Chem Soc 130:14802-14812. https ://doi.org/10.1021/ja8054832

Kerfah R, Plevin MJ, Sounier R, Gans P, Boisbouvier J (2015) Methylspecific isotopic labeling: a molecular tool box for solution NMR studies of large proteins. Curr Opin Struct Biol 32:113-122. https ://doi.org/10.1016/j.sbi.2015.03.009

Lescanne $\mathrm{M}$ et al (2017) Methyl group assignment using pseudocontact shifts with PARAssign. J Biomol NMR. 69(4):183-195.
Li J et al (2012) Structure insights into mechanisms of ATP hydrolysis and the activation of human heat-shock protein 90. Acta Biochim Biophys Sin 44:300-306. https://doi.org/10.1093/abbs/gms001

Liu WM, Overhand M, Ubbink M (2014) The application of paramagnetic lanthanoid ions in NMR spectroscopy on proteins. Coord Chem Rev 273:2-12. https://doi.org/10.1016/j.ccr.2013.10.018

Murray CW et al (2010) Fragment-based drug discovery applied to Hsp90. Discovery of two lead series with high ligand efficiency. J Med Chem 53:5942-5955. https://doi.org/10.1021/jm100059d

Nagaraju GP et al (2017) Epigenetic effects of inhibition of heat shock protein 90 (HSP90) in human pancreatic and colon cancer. Cancer Lett 402:110-116. https://doi.org/10.1016/j.canlet.2017.05.021

Otting G (2010) Protein NMR using paramagnetic ions. Annu Rev Biophys Biomol Struct 39:387-405. https://doi.org/10.1146/annur ev.biophys.093008.131321

Palmer AG, Cavanagh J, Wright PE, Rance M (1991) Sensitivity improvement in proton-detected 2-dimensional heteronuclear correlation Nmr-spectroscopy. J Magn Reson 93:151-170. https ://doi.org/10.1016/0022-2364(91)90036-S

Pellecchia $\mathrm{M}$ et al (2008) Perspectives on NMR in drug discovery: a technique comes of age. Nat Rev Drug Discov 7:738-745. https ://doi.org/10.1038/nrd2606

Saio $\mathrm{T}$ et al (2011) An NMR strategy for fragment-based ligand screening utilizing a paramagnetic lanthanide probe. J Biomol NMR 51:395-408. https://doi.org/10.1007/s10858-011-9566-5

Saio T, Guan X, Rossi P, Economou A, Kalodimos CG (2014) Structural basis for protein antiaggregation activity of the trigger factor chaperone. Science 344:1250494. https://doi.org/10.1126/scien ce. 1250494

Sattler M, Fesik SW (1997) Resolving resonance overlap in the NMR spectra of proteins from differential lanthanide-induced shifts. J Am Chem Soc 119:7885-7886. https://doi.org/10.1021/ja971 $356 \mathrm{~m}$

Schleucher J et al (1994) A general enhancement scheme in heteronuclear multidimensional NMR employing pulsed field gradients. J Biomol NMR 4:301-306. https://doi.org/10.1007/BF00175254

Skinner SP, Moshev M, Hass MA, Keizers PH, Ubbink M (2013) PARAssign-paramagnetic NMR assignments of protein nuclei on the basis of pseudocontact shifts. J Biomol NMR 55:379-389. https ://doi.org/10.1007/s10858-013-9722-1

Sprangers R et al (2008) TROSY-based NMR evidence for a novel class of $20 \mathrm{~S}$ proteasome inhibitors. Biochemistry 47:6727-6734. https ://doi.org/10.1021/bi8005913

Tu K, Gochin M (1999) Structure determination by restrained molecular dynamics using NMR pseudocontact shifts as experimentally determined constraints. J Am Chem Soc 121:9276-9285. https:// doi.org/10.1021/ja9904540

Tugarinov V, Kay LE (2005) Methyl groups as probes of structure and dynamics in NMR studies of high-molecular-weight proteins. Chembiochem 6:1567-1577. https://doi.org/10.1002/cbic.200500110

Tugarinov V, Hwang PM, Ollerenshaw JE, Kay LE (2003) Crosscorrelated relaxation enhanced $1 \mathrm{H}-13 \mathrm{C}$ NMR spectroscopy of methyl groups in very high molecular weight proteins and protein complexes. J Am Chem Soc 125:10420-10428. https://doi. org/10.1021/ja030153x

Viegas A, Manso J, Nobrega FL, Cabrita EJ (2011) Saturation-transfer difference (STD) NMR: a simple and fast method for ligand screening and characterization of protein binding. J Chem Educ 88:990-994. https://doi.org/10.1021/ed101169t

Waudby CA, Ramos A, Cabrita LD, Christodoulou J (2016) Twodimensional NMR lineshape analysis. Sci Rep. https://doi. org/10.1038/srep24826

Wiesnerl S, Sprangers R (2015) Methyl groups as NMR probes for biomolecular interactions. Curr Opin Struct Biol 35:60-67. https ://doi.org/10.1016/j.sbi.2015.08.010 